\title{
Heat Set Creases in Polyethylene Terephthalate (PET)Sheets to Enable Origami-Based Applications
}

\author{
Larry L. Howell \\ Brigham Young University - Utah, Ihowell@byu.edu \\ Nathan C. Brown \\ Brigham Young University, nathancbrown94@gmail.com \\ Brandon S. Sargent \\ Brigham Young University, bsargent@byu.net \\ Brian D. Jensen \\ Brigham Young University, bdjensen@byu.edu \\ Spencer P. Magleby \\ Brigham Young University, magleby@byu.edu
}

See next page for additional authors

Follow this and additional works at: https://scholarsarchive.byu.edu/data

Part of the Engineering Commons

\section{BYU ScholarsArchive Citation}

Howell, Larry L.; Brown, Nathan C.; Sargent, Brandon S.; Jensen, Brian D.; Magleby, Spencer P.; and Pitt, William G., "Heat Set Creases in Polyethylene Terephthalate (PET)Sheets to Enable Origami-Based Applications" (2019). ScholarsArchive Data. 8.

https://scholarsarchive.byu.edu/data/8

This Data is brought to you for free and open access by BYU ScholarsArchive. It has been accepted for inclusion in ScholarsArchive Data by an authorized administrator of BYU ScholarsArchive. For more information, please contact scholarsarchive@byu.edu, ellen_amatangelo@byu.edu. 


\section{Authors}

Larry L. Howell, Nathan C. Brown, Brandon S. Sargent, Brian D. Jensen, Spencer P. Magleby, and William G. Pitt 


\section{Data Set for "Heat Set Creases in Polyethylene Terephthalate (PET) Sheets to Enable Origami-Based Applications"}

This data set includes the raw data from the tests described in the research paper "Heat Set Creases in Polyethylene Terephthalate (PET) Sheets to Enable Origami-Based Applications" by Brandon Sargent, Nathan Brown, Brian D. Jensen, Spencer P. Magleby, William G. Pitt, and Larry L. Howell.

\section{Annealing Data}

\section{Folder Navigation:}

Annealing data is found in the folder named "Annealing Data." Each folder inside this folder is named as to signify the sample numbers it contains. Each folder contains the corresponding amount of raw data files labeled beginning counting from one. The first raw data file in the folder corresponds to the first sample number in the file name. For example, folder name "s301-308.is_tens_RawData" contains data for samples 301-308. Inside that folder contains files beginning with "Specimen_RawData_1" which corresponds to sample 301. "Specimen_RawData_2" would then correspond to sample 302, and so forth.

\section{Sample Overview:}

Below is an overview of the sample numbers used in each testing condition set.

\begin{tabular}{|c|c|c|c|}
\hline $\begin{array}{c}\text { Annealing } \\
\text { Temperature }\left({ }^{\circ} \mathrm{C}\right) \\
\end{array}$ & $\begin{array}{c}\text { Anneal Time } \\
\text { (min) }\end{array}$ & Cooling Rate & Sample Numbers \\
\hline 120 & 60 & Drop & $441-450,996-1000$ \\
\hline 120 & 75 & Drop & $432-440,991-995$ \\
\hline 120 & 90 & Drop & $421-430,986-990$ \\
\hline 120 & 105 & Drop & $411-420,981-985$ \\
\hline 120 & 120 & Drop & $401-410,976-980$ \\
\hline 120 & 60 & Slow Cool & $491-500,1021-1025$ \\
\hline 120 & 75 & Slow Cool & $481-490,1026-1030$ \\
\hline 120 & 90 & Slow Cool & $471-480,1031-1035$ \\
\hline 120 & 105 & Slow Cool & $461-470,1036-1040$ \\
\hline 120 & 120 & Slow Cool & $451-460,1041-1045$ \\
\hline 130 & 60 & Drop & $391-400,951-955$ \\
\hline 130 & 75 & Drop & $381-390,956-960$ \\
\hline 130 & 90 & Drop & $371-380,961-965$ \\
\hline 130 & 105 & Drop & $361-370,966-970$ \\
\hline 130 & 120 & Drop & $351-360,971-975$ \\
\hline 130 & 60 & Slow Cool & $341-350$ \\
\hline 130 & 75 & Slow Cool & $331-340$ \\
\hline 130 & 90 & Slow Cool & $321-330$ \\
\hline
\end{tabular}




\begin{tabular}{|c|c|c|c|}
\hline 130 & 105 & Slow Cool & $311-320$ \\
\hline 130 & 120 & Slow Cool & $301-310$ \\
\hline 140 & 60 & Drop & $241-250,941-949$ \\
\hline 140 & 75 & Drop & $231-240,931-940$ \\
\hline 140 & 90 & Drop & $221-230,921-930$ \\
\hline 140 & 105 & Drop & 211-220, 911-920 \\
\hline 140 & 120 & Drop & $201-210,901-910$ \\
\hline 140 & 60 & Slow Cool & $291-300$ \\
\hline 140 & 75 & Slow Cool & $281-290$ \\
\hline 140 & 90 & Slow Cool & $271-280$ \\
\hline 140 & 105 & Slow Cool & $261-270$ \\
\hline 140 & 120 & Slow Cool & $251-260$ \\
\hline 150 & 60 & Drop & $91-100,737-745$ \\
\hline 150 & 75 & Drop & $81-90,728-736$ \\
\hline 150 & 90 & Drop & $71-80,719-727$ \\
\hline 150 & 105 & Drop & $61-70,710-718$ \\
\hline 150 & 120 & Drop & 51-60, 701-709 \\
\hline 150 & 60 & Slow Cool & $41-50,831-840$ \\
\hline 150 & 75 & Slow Cool & $31-40,841-850$ \\
\hline 150 & 90 & Slow Cool & $21-30,851-860$ \\
\hline 150 & 105 & Slow Cool & $11-20,861-870$ \\
\hline 150 & 120 & Slow Cool & $1-10,871-880$ \\
\hline 160 & 60 & Drop & $191-200$ \\
\hline 160 & 75 & Drop & $181-190$ \\
\hline 160 & 90 & Drop & $171-180,641-650$ \\
\hline 160 & 105 & Drop & $161-170,661-680$ \\
\hline 160 & 120 & Drop & $151-160,681-700$ \\
\hline 160 & 60 & Slow Cool & $141-150,751-760$ \\
\hline 160 & 75 & Slow Cool & $131-140,761-770$ \\
\hline 160 & 90 & Slow Cool & $121-130,771-780$ \\
\hline 160 & 105 & Slow Cool & $111-120,781-790$ \\
\hline 160 & 120 & Slow Cool & $101-110,791-800$ \\
\hline 170 & 60 & Drop & $501-510$ \\
\hline 170 & 75 & Drop & $511-520$ \\
\hline 170 & 90 & Drop & $521-530$ \\
\hline 170 & 105 & Drop & $531-540$ \\
\hline 170 & 120 & Drop & $541-550$ \\
\hline 170 & 60 & Slow Cool & $551-560$ \\
\hline 170 & 75 & Slow Cool & $561-570$ \\
\hline 170 & 90 & Slow Cool & $571-580$ \\
\hline 170 & 105 & Slow Cool & $581-590$ \\
\hline
\end{tabular}




\begin{tabular}{|l|c|c|l|}
\hline 170 & 120 & Slow Cool & $591-600$ \\
\hline 180 & 60 & Drop & $601-605,611-620$ \\
\hline 190 & 60 & Drop & $606-610,651-660$ \\
\hline 200 & 60 & Drop & $881-895$ \\
\hline 200 & 60 & Drop & $621-635,1017-1020$ \\
\hline 200 & 75 & Drop & $1013-1016$ \\
\hline 200 & 90 & Drop & $1009-1012$ \\
\hline 200 & 105 & Drop & $1005-1008$ \\
\hline 200 & 120 & Drop & $1001-1004$ \\
\hline 210 & 60 & Drop & $816-830$ \\
\hline 210 & 60 & Drop & $801-815$ \\
\hline 210 & 60 & Drop & $1046-1050$ \\
\hline 210 & 90 & Drop & $1051-1055$ \\
\hline
\end{tabular}

\section{Optical Absorption Data}

\section{Sample Overview:}

The spreadsheet file named "Sample Overview_Absorption.xlsx" reports the selected samples from each testing condition set and their corresponding optical absorption values.

\section{Summary Files}

The folder designated "Summary Files" contains the data in an easy to analyze format. These files were used for the statistics cited in the paper. Due to the straightforward reporting of data values, these files will most likely be the most helpful to researchers. Some samples were removed due to physical conditions that made the data for the test unusable. Note that statistical outliers have not been removed from these data sets.

Below are the names of the files followed by an explanation of its contents:

\section{ForceMax_Complete}

This reports the max force for every sample reported with the corresponding testing conditions: annealing temperature, anneal time and cooling rate (0 represents drop cool condition, 1 represents slow cool condition). This collection includes data from duplicate tests.

\section{ForceMax_Refined}

This reports the max force for every sample, reported with the corresponding testing conditions: annealing temperature, anneal time and cooling rate (0 represents drop cool condition, 1 represents slow cool condition). This file does not contain duplicate test data but includes data from last test of each testing condition. 


\section{ForceDrop_Complete}

This reports the force drop observed in the stress relaxation tests, including data from duplicate tests. These values are reported with the corresponding testing conditions: annealing temperature, anneal time and cooling rate ( 0 represents drop cool condition, 1 represents slow cool condition).

\section{ForceDrop_Refined}

This reports the force drop observed in the stress relaxation tests. These values are reported with the corresponding testing conditions: annealing temperature, anneal time and cooling rate ( 0 represents drop cool condition, 1 represents slow cool condition). While "ForceDrop_Complete" includes data from duplicate tests, this file only contains data from the last test of each testing condition.

\section{Absorption_Force}

This file reports the absorption data from tested sample in two sections. The first section is a complete set of the testing data, including duplicate tests. The second section is a refined data set that includes the last samples tested from each testing condition. 